\title{
Early cellular and molecular changes induced by diabetes in the retina
}

\author{
M. Lorenzi, C. Gerhardinger \\ Schepens Eye Research Institute and Department of Ophthalmology, Harvard Medical School, Boston, Massachussetts
}

\section{Abstract}

For several decades the pathobiology of diabetic retinopathy has been the object of conjecture and hypotheses. Indeed, very little was known about the cellular events triggered by diabetes in the retina and about the processes underlying the microangiopathy. In the last few years there has been a concerted effort to acquire such information, and the work has targeted not just the retinal vessels but more comprehensively the retina. The picture emerging is one of multiplicity: multiple cell types in the retina are affected early by diabetes and multiple processes are opera- tive in the microangiopathy. The main abnormalities captured to date are altered expression of several genes, apoptosis, microthrombosis, and proinflammatory changes. The new information needs to be integrated into temporal and mechanistic sequences and further expanded but it is beginning to provide a framework for the cellular dimension of diabetic retinopathy. [Diabetologia (2001) 44: 791-804]

Keywords Diabetic retinopathy, neural retina, glia, pericytes, endothelial cells, apoptosis, thrombosis, inflammation, human diabetes, streptozotocin-diabetic rat
Important knowledge has been gained during the last decade on how to delay the onset and progression of diabetic retinopathy by primary treatment of the underlying diabetic state. The value of tight glycaemic control, strongly suggested by the pioneer prospective study of Pirart [1], has been unequivocally affirmed by the randomized Diabetes Control and Complication Trial (DCCT) and the United Kingdom Prospective Diabetes Study (UKPDS). In addition, ongoing follow-up of the DCCT cohorts is demonstrating that protection from progressive retino-

Received: 24 October 2000 and in revised form: 16 March 2001

Corresponding author: M.Lorenzi, M.D., Schepens Eye Research Institute, 20 Staniford St., Boston, Mass., 02114, U.S.A., e-mail: lorenzi@vision.eri.harvard.eda

Abbreviations: AGE, Advanced glycation end-products; GFAP, glial fibrillary acidic protein; GLUT 1, glucose transporter 1; ICAM-1, intercellular adhesion molecule-1; IGF-1, insulin-like growth factor-1; nNOS, neuronal nitric oxide synthase; PKC, protein kinase C; VEGF, vascular endothelial growth factor. pathy persists for years in the group treated with intensive therapy, despite increasing hyperglycaemia [2]. By showing the added value of hypertension control [3], the UKPDS has launched the era of combined approaches to prevent or delay diabetic retinopathy. The next target or set of targets to be addressed therapeutically should become clearer as our understanding of the pathogenetic process deepens. The availability of new technology has recently moved the focus of investigation from histology and biochemistry to the cellular and molecular levels. This new investigative mode is bound to generate more questions than answers for some time because it must go through a descriptive phase centered on cells and molecules of a complex organ, which will bring to the fore multiple and diverse abnormalities whose respective roles have to be established. We write this review in the midst of such a descriptive phase. The focus will be on changes relevant to early non-proliferative retinopathy, and especially on those changes that generate new themes and are verified or found in the human retina. We will confine our re- 


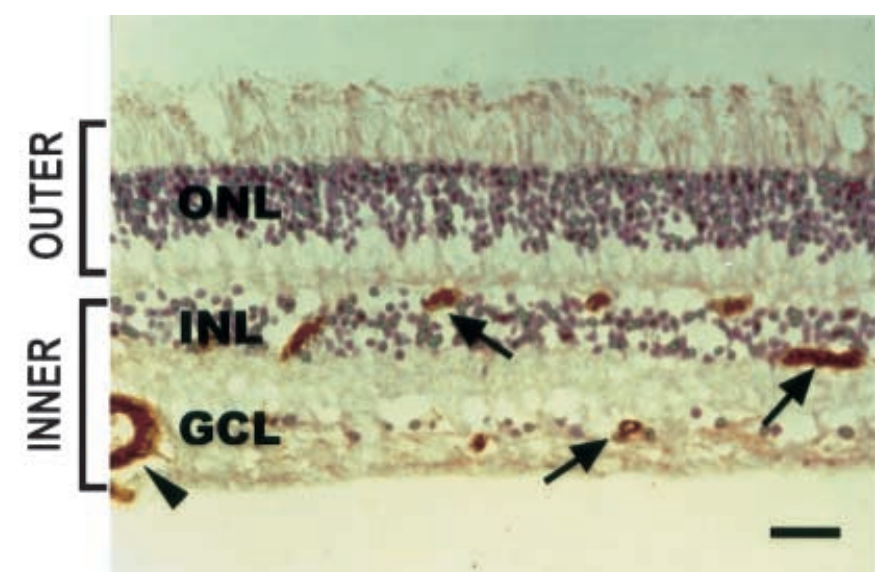

Fig. 1. Radial section of human retina immunostained for von Willebrand factor to identify the vessels and counterstained with haematoxylin and eosin to show the nuclear layers. Vessels (brown) are confined to the inner retina. Arrows point to small vessels sectioned tangentially or radially; the arrowhead points to a large vessel ONL, outer nuclear layer; INL, inner nuclear layer; GCL, ganglion cell layer. Scale bar $40 \mu \mathrm{m}$

view and discussion to observations made in the retina in situ because these are the foundation on which to reconstruct the processes operative in diabetic retinopathy, and cite only occasionally the contributions derived from studies in cultured cells.

\section{Multiple cell types in the retina are affected by diabetes}

The characteristic and clinically best known feature of non-proliferative diabetic retinopathy is microangiopathy that develops almost universally in patients who have had Type I (insulin-dependent) or Type II (non-insulin-dependent) diabetes mellitus 15 or more years [4, 5]. Microangiopathy threatens sight when it leads to macular edema and/or retinal ischaemia with attendant unregulated angiogenesis. Macular edema develops when abnormal permeability of retinal capillaries causes the passive influx of plasma or blood into the retina which overwhelms the active reabsorbing transport. Retinal ischaemia develops when a critical number of capillaries becomes nonperfused and obliterated. The simplest paradigm to explain capillary permeability and closure centres on vascular endothelium. In the retina, as in the brain, endothelial cells are the site of the blood-tissue barrier, and, as in all vessels, provide a non-thrombogenic surface for blood flow. Both these properties are eventually compromised by diabetes.

On the other hand, diabetes also affects the neural and glial cells of the retina. This is not surprising given that diabetes impacts on most cell types in the body, but it is potentially very important because the neural and glial cells appear to be affected very early on in the course of diabetes, and could thus claim a causative or contributory role in the microangiopathy. Reports of electroretinographic changes in diabetic patients without demonstrable vascular lesions date back to the 1960s [6] and have been confirmed by several authors, who found the electroretinographic abnormalities to originate in the ganglion and inner nuclear layers $[7,8]$. Studies mostly performed in streptozotocin-induced diabetic rats have now identified specific molecular changes of neuroglial elements in these retinal layers very early after induction of diabetes. The inner two-thirds of the retina, in which the retinal capillaries reside (Fig.1) host close to 50 different cell types ([9] and R. Masland, personal communication), intimately interdependent in physiology, and likely therefore to influence one another in pathological circumstances. An example of the importance of such reciprocal influences is provided by retinitis pigmentosa, in which the genetic defects affect rod photoreceptors, but it is the subsequent slow degeneration of cones that is most important for visual loss [10]. Whether the neuroglial abnormalities induced by diabetes eventually contribute to the development of vascular pathology is not known at present, but the neuroglial abnormalities will be presented first in view of their early appearance and potential impact on some aspects of the microangiopathy.

Abnormalities of the neural retina in experimental diabetes. In the rat retina, most of the neurons containing neuronal nitric oxide synthase (nNOS) appear to be amacrine cells, residing in the inner nuclear layer or displaced to the ganglion cell layer and closely related to vessels $[11,12]$. The number of these nNOScontaining cells was found to decrease by $32 \%$ as early as 1 week after the induction of streptozotocin diabetes and remained reduced for up to 8 months [12]. The study did not, however, address the question whether the reduced number of nNOS-positive cells was due to reduced synthesis of the protein or to death of the cells. Reduced availability of a vasodilator such as nitric oxide could contribute to the reduced retinal blood flow reported after a short duration of experimental diabetes and so compound the vascular effects of increased retinal endothelin levels. Increased endothelin-1 [13,14] and endothelin-3 [13] immunostaining has been observed in the neural cells of the inner retina 2-4 weeks after the induction of streptozotocin diabetes. Even though immunohistochemical studies are not able to give precise information about the synthesis of a secreted protein, the observations are in agreement with several reports of increased endothelin mRNAs in the retina of diabetic rats $[13,15,16]$. The expression of endothelin receptors, detected in all retina layers as well as on vascular cells, appeared to decrease after 2 weeks of streptozotocin diabetes [14] but increase thereafter [13-15]. Studies with inhibitors of endothelin action $[13,16]$ 
or synthesis [16] have implicated this vasoconstrictor peptide in the causation of reduced retinal blood flow, although it is noteworthy that reduced blood flow was no longer detectable after six months of diabetes in rats with a persistent increase in retinal endothelin expression [13]. A possible explanation suggested by in vitro studies [17] is the development of postreceptor resistance to endothelin action caused by chronic hyperglycaemia. A general endothelin receptor antagonist has recently been reported to partially prevent basement membrane thickening in the retinal capillaries of diabetic rats [18], an outcome that could reflect the prevention of blood flow changes or more direct actions of endothelin on the expression of extracellular matrix components [18]. It has been suggested that the activation of protein kinase $\mathrm{C}$ (PKC) induced by high glucose is the mechanism for the increased endothelin observed in the diabetic rat retina [16]. The $\mathrm{PKC}$ is activated in the retina of streptozotocin-diabetic rats [19] and a selective inhibitor of the $\beta 2$ isoform of PKC was shown to reduce the increased mean retinal circulation time observed in this model [19]. However, the effects of the inhibitor on retinal endothelin were not measured. This information, and information on which cell types in the retina show activation of $\mathrm{PKC}$ in diabetes, would help show whether $\mathrm{PKC}$ and endothelin are linked in the causation of blood flow abnormalities or not.

Early in the course of streptozotocin diabetes (2-8 weeks) there is down regulation of retinal GLUT 1 expression [20]. GLUT 1 is the ubiquitous glucose transporter isoform that is not regulated by insulin and is expressed in the retina by ganglion cells, photoreceptors, and Müller glial cells, in addition to the vascular cells [21]. To determine GLUT 1 expression after a longer duration of diabetes will be interesting, in view of the fact that GLUT 1 expression as well as hexose transport are regulated by vascular endothelial growth factor (VEGF), which is reported to increase in the retina after several months of streptozotocin diabetes $[22,24]$. Although VEGF immunoreactivity was not altered after 2 [25] or 3 [23] months of diabetes, immunoreactive VEGF [23, 24], its transcript [24] and the transcripts encoding the high-affinity VEGF receptors flk-1 and flt-1 [24] were found to increase in the inner as well as the outer retina of rats who had diabetes for 6 months. This time-course suggests that retinal VEGF up-regulation in experimental diabetes is not the immediate consequence of the prevailing metabolic abnormalities but could rather reflect a changing environment in the retina. The main implication of VEGF overexpression is that it could mediate the compromised barrier properties of diabetic retinal vessels. VEGF is a powerful permeability factor [26], exogenously administered VEGF induces retinal edema [27], and a spatial correlation has been noted in the rat retina between the appearance of VEGF immunoreactivity and albumin extravasation [23]. However, increases in vascular permeability have been reported in the rat retina as early as 2 weeks [28] or 3 months [29] after the induction of diabetes, within a period when increased VEGF expression is not yet to be expected, at least on the basis of available studies [23, 25].

Perhaps the most dramatic abnormality occurring early in the neural retina of diabetic rats is a ten-fold increase in the frequency of apoptosis, observed after only 1 month of streptozotocin-induced diabetes and continuing with the same frequency for at least 12 months [30]. The vast majority of apoptotic cells were not found in association with vascular markers and appeared to be ganglion cells. Although such a substantial increase in the rate of apoptosis of postmitotic cells might seem incompatible with the longterm integrity of the retina, the calculated numerical cell loss over several months of experimental diabetes was consistent with measured morphometric changes which would not be detected clinically [30]. The occurrence of both neural [30] and vascular [31,32] apoptosis in the diabetic retina suggests a compromised availability of survival signals. In this context, a recent study has addressed expression and signaling of insulin-like growth factor 1 (IGF-1) in experimental diabetic retinopathy [33]. The IGF-1 is a most potent growth/survival factor for various cell types, including neural and vascular [34,35], both IGF-1 and its receptor are present in the retina [36], and circulating IGF-1 levels as well as IGF-1 expression in several tissues are greatly reduced in both human and experimental diabetes [37]. Retinal IGF-1 mRNA levels were not found to change after 2 months of streptozotocin-induced diabetes, but after 5 months of diabetes they were only half the levels recorded in control rats matched for age [33]. The suppressed IGF-1 synthesis was not due to the reduced growth hormone levels and action characteristic of the streptozotocin-diabetic rat [38], because the study of hypophysectomized rats showed that retinal IGF-1 synthesis is growth hormone-independent [33]. Surprisingly, neither the levels of IGF-1 receptor phosphorylation nor the phosphorylation of Akt, the main downstream anti-apoptotic effector of IGF-1 [34] were found to decrease in the diabetic rats, despite the suppressed IGF-1 synthesis. This is noteworthy for the additional reason that Akt phosphorylation is also downstream of the insulin receptor [34], and the diabetic rats in the study had systemic signs of insulin deficiency. It thus appears that in the retina of diabetic rats, the activation of the IGF-1 receptor is modulated by influences that compensate for, or are compensated by, suppressed IGF-1 synthesis and that decreased signalling through the IGF-1 receptor cannot readily be invoked to explain accelerated apoptosis in the diabetic retina.

Abnormalities of the neural retina in human diabetes. Neural abnormalities induced by diabetes have also 
been found in the human retina, although the list is shorter and the extent of abnormalities less conspicuous than in the streptozotocin-diabetic rat. There are no reports to our knowledge on the behavior of nNOS-containing neurons or expression of endothelin in the human diabetic retina. The distribution and intensity of GLUT 1 immunostaining were found to be virtually identical in the postmortem retinas of diabetic and nondiabetic donors [39]. However, the study was not quantitative because GLUT 1 was evaluated solely by immunohistochemistry without confirmatory measurement of protein abundance. Several groups of investigators have addressed the behaviour of VEGF in human non-proliferative retinopathy, and although the findings are not in full agreement, their aggregate does not confirm the dramatic VEGF increase described in the neural retina of diabetic rats [24]. VEGF was found to be constitutively expressed in the adult human (and rat) retina, being synthesized in the inner nuclear and ganglion cell layers, and present, in addition, in the synaptic terminals of photoreceptors and the wall of blood vessels [40]. In this study, the combined results of quantitative Northern blot analysis, RT-PCR (to examine the splicing pattern of the VEGF transcript), in situ hybridization, and immunohistochemistry failed to identify differences in VEGF abundance or distribution between the retinas of diabetic subjects with early nonproliferative retinopathy and the retinas of non-diabetic subjects [40]. Other authors did not detect constitutive presence of VEGF in the non-diabetic human retina but reported the appearance of VEGF immunostaining in Müller glial cells [41] and vessels [42, 43 ] of diabetic retinas. The controversy over constitutive retinal expression of VEGF appears to be resolving, because the very investigators who had initially reported undetectable VEGF mRNA in the nonischaemic monkey retina [44], have lately discovered abundant constitutive expression of VEGF in such normal monkey (and rat) retinas [45]. The significance of the increased VEGF immunostaining around diabetic retinal vessels is not easy to assess, because immunostaining cannot throw light on synthesis, especially of a secreted protein, and, in addition, VEGF is present in the systemic circulation. In other words, increased VEGF staining around diabetic retinal vessels showing evidence of leakage [23, 42] could simply reflect VEGF extravasation and cumulative binding to heparin groups in the basement membranes.

Furthermore, the phenomenon of early neuroretinal apoptosis appears to be less prominent in human diabetes than in the streptozotocin-diabetic rat. An initial study found no evidence of neural apoptosis in the retina of seven diabetic donors with early nonproliferative retinopathy [31], but only one retinal section was examined for each donor, a sample that could have been too small to detect a rapidly consum- mated phenomenon such as apoptosis. The use of flat mounts of retinal quadrants has provided larger fields of observation and led to the detection of increased neural apoptosis in diabetic donors [30]. However, the study compared only three non-diabetic and two diabetic donors, and one of the latter had a 30-year history of diabetes with established retinopathy. In the retina of the other diabetic donor, who had a 6year history of diabetes and no clinical retinopathy, the number of apoptotic neural cells was twofold greater than in the non-diabetic specimens. Overall, it is likely that neural retinal cells undergo accelerated apoptosis in diabetes, and that the process explains the extensive atrophy of retinal neurons and fibres observed in patients with advanced diabetic retinopathy. However, the issues of how early and extensive neural apoptosis is in human diabetes, and whether it precedes microangiopathy are still to be settled. Likewise, it is not known whether the greatly suppressed IGF-1 synthesis observed in the retinas of human diabetic subjects [33] plays a role in neural apoptosis. The postmortem period, which did not affect the stability of the IGF-1 transcript, resulted in complete loss of tyrosine phosphorylation of the IGF-1 receptor [33], thus precluding studies of receptor activation. The data obtained in diabetic rats [33] and discussed earlier indicate that reduced IGF-1 synthesis might not translate into reduced signalling, but the actual events in the human diabetic retina are not currently known.

Abnormalities of retinal glial cells in experimental and human diabetes. The mammalian retina contains two types of macroglial cells: Müller cells that are specific to the retina, and astrocytes which migrate into the retina from the optic nerve [46]. Müller cells extend their radial processes to span the thickness of the retina (Fig. 2), while astrocytes are confined to the nerve fiber layer. In their respective locations, the two cell types envelope neurons, the initial segments of the ganglion cell axons, and blood vessels. Specifically, the inner layer of retinal capillaries is enveloped by both astrocytic and Müller cell processes, while only the latter provide the glial wrapping to the outer layer of the retinal vasculature [46]. Glial processes are so inextricably enmeshed in the outer basement membranes of retinal microvessels, that it is practically impossible to isolate retinal capillaries free of glial contamination [47].

Müller cells have characteristics that make them both potential targets of diabetes and potential contributors to retinopathy. Akin to brain astrocytes, Müller cells are the primary site of glucose uptake and phosphorylation in the retina [48]. They are endowed with GLUT 1 [39], which permits unregulated transport of glucose. They metabolize glucose intensely through glycolysis to produce lactates that fuel neuronal metabolism [48], are the primary site of gly- 


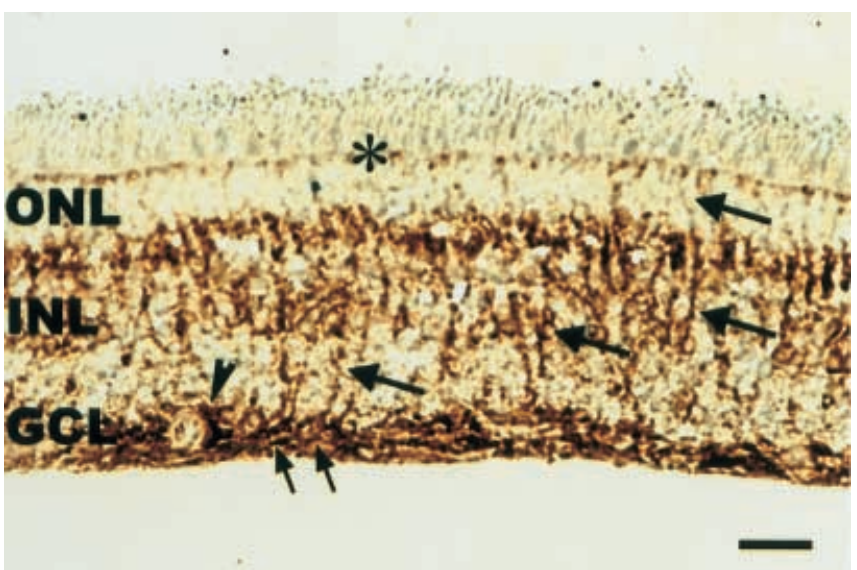

Fig. 2. Radial section of human retina immunostained for Bcl2 to identify Müller cells. In the adult retina Bcl-2 is confined to Müller cells. The small arrows point to Müller cell endfeet; the arrowhead to Müller cell processes investing a large vessel; and the arrows to the radial processes spanning the thickness of the retina, and contributing to the outer limiting membrane $(*)$. The wall of the vessel does not show Bcl-2 immunostaining. Scale bar $40 \mu \mathrm{m}$

cogen storage and metabolism in the retina [48] and also have aldose reductase $[49,50]$, which exposes them to the consequences of polyol pathway activation. Some of the Müller cells housekeeping functions are linked to the regulation of retinal blood flow. These cells express several ion channels and co-transporters responsible for the rapid removal of potassium ions, $\mathrm{CO}_{2}$, and other metabolites released by neurons in the synaptic space [51]. The subsequent release of potassium and acid equivalents at the endfeet of Müller cells (known as the Müller cell "spatial buffering system") is thought to be the mechanism coupling neuronal activity to blood flow regulation [51]. Being the only cells in the retina endowed with the enzyme glutamine synthetase [51], Müller cells transform the glutamate taken up via high-affinity carriers into glutamine, which is then returned to the neural cells for glutamate resynthesis [48]. Finally, in the retina as in the brain, the acquisition of barrier properties by vascular endothelial cells appears to be driven by the surrounding neuro-glial environment [52], and Müller cells share with astrocytes the capacity to induce barrier characteristics in endothelial cells [53].

Until recently, retinal glia was known to be involved only in the late stages of diabetic retinopathy. The B-wave of the electroretinogram, which originates from Müller cells, shows reduced amplitude in advanced or proliferative retinopathy [7], and glial cell invasion is probably the final chapter in the obliterative process of microvessels [54]. There is now evidence that retinal glia, and Müller cells in particular, are affected early in the course of both experimental and human diabetes. In the context of various retinal pathologies, a recurrent pattern of Müller cell abnormalities includes increased expression of the anti-apoptotic molecule Bcl-2 and of the intermediate filament protein glial fibrillary acidic protein (GFAP), together with reduced expression of the enzyme glutamine synthetase [55]. In the rat, marked overexpression of GFAP was observed as early as 3 months after the induction of streptozotocin diabetes [56]. The investigators also detected a reduced ability to convert glutamate into glutamine and increased glutamate in the diabetic rat retinas, and proposed that glutamate excitotoxicity occurs in the diabetic retina as a consequence of Müller cell dysfunction [56]. The increased GFAP appears to occur mainly in Müller cells [28, 57], with astrocytes showing instead reduced GFAP immunoreactivity after 2 months of streptozotocin diabetes [57] and possibly a reduced number [28]. Of note, Müller cell hyperplasia but not yet increased GFAP expression has been found after 4 weeks of diabetes [28], suggesting that the latter does not merely reflect increased Müller cell numbers. In one of the studies [28], all glial abnormalities were preceded by an increase in retinal capillary permeability detected as a leakage of intravascularly injected Evans blue after only 2 weeks duration of experimental diabetes. The authors thus proposed that glial abnormalities in the diabetic retina are a consequence of vascular leakage. This is a distinct possibility which requires confirmation.

The retinas of human donors with early diabetic microangiopathy showed a selective and prominent increase in GFAP [55]. The typical Müller cell pattern of Bcl-2 and glutamine synthetase immunostaining was similar for both intensity and distribution in the diabetic and non-diabetic retinas, as were quantitatively the levels of the two proteins. In contrast, GFAP, largely confined to the most proximal retina in the non-diabetic donors, was present in most diabetic retinas along the entire length of the Müller cell processes, throughout the outer retina. Accordingly, the GFAP content was increased three-fold in the diabetic retinas [55]. Insofar as the content of Bcl-2 and glutamine synthetase did not change, the overexpression of GFAP appears selective and is not likely to reflect an increased number of Müller cells. Thus, in both human and experimental diabetes, the circumstances of increased retinal GFAP point to altered regulation of gene expression. This could be because of selective transcriptional effects of high glucose or other metabolic abnormalities on the GFAP gene, or be an element of more pervasive changes in Müller cells yielding a "reactive" phenotype.

Even as an isolated abnormality, increased GFAP could have consequences because transgenic mice overexpressing GFAP show a phenotype that ranges from early postnatal lethality to degenerative changes in the central and peripheral nervous system, in direct relation to transgene expression [58]. If GFAP 
Table 1. Changes in the cells of retinal vessels in early diabetic retinopathy ${ }^{\mathrm{a}}$

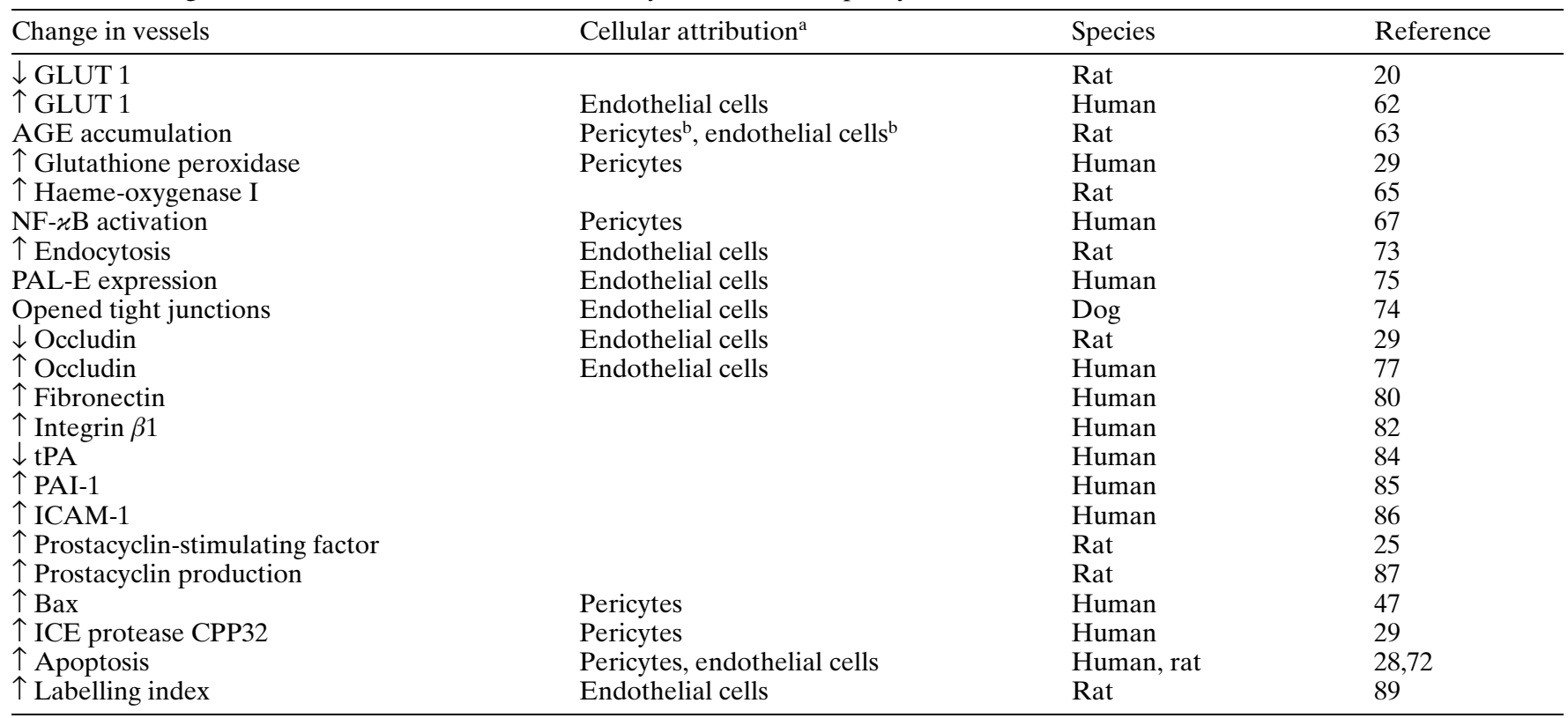

${ }^{a}$ Assigned when permitted by the detection method used or on the basis of known selectivity of expression

${ }^{\mathrm{b}}$ Accumulation could be in basement membrane apposed to cells

up regulation is a marker of a "reactive" phenotype, an even more complex set of consequences could reasonably be envisioned. Best studied in relation to events in the central nervous system, reactive glia up-regulates the expression of a multitude of molecules, including molecules involved in cell adhesion and migration, tissue repair and scar formation, inflammation and neuro protection [59]. Such an altered local environment would magnify tremendously the number of abnormal signals received by retinal vascular cells early in the course of diabetes and potentially impact on their regulation of blood flow, barrier properties, and even survival.

\section{Abnormalities of retinal vascular cells in experimental and human diabetes. Cellular and molecular studies of retinal vessels in diabetes have focused on identify- ing mechanisms for glucose toxicity, altered proper- ties of the endothelial barrier, and capillary oblitera- tion. Table 1 summarizes the changes described to date in diabetic retinal vessels in situ or in vessels iso- lated from the fresh retina and indicates the cell type exhibiting the abnormality when the attribution was unequivocal.}

Changes relevant to glucose toxicity. The expression of GLUT 1 has been found to be reduced in the retinal vessels of rats that have had streptozotocin diabetes for 2 months [20], and previous studies in rats with similarly short duration of diabetes had shown increased $\mathrm{K}_{\mathrm{m}}$ values for the glucose transport systems of the blood-retinal barrier [60]. While these changes could be directed at protecting vascular cells as well as the neural retina from the effects of higher blood glucose, in vitro data indicate that down-regulation of glucose transport by high glucose concentrations does not suffice to achieve normalization of intracellular glucose concentrations in the presence of sustained high glucose [61]. It is thus not certain whether changes in GLUT 1 can be expected to modulate significantly the impact of hyperglycaemia on retinal cells. Moreover, preliminary studies comparing retinal microvessels of three diabetic and two non-diabetic human donors have shown a bimodal distribution of GLUT 1, with the larger proportion of diabetic microvessels manifesting a dramatic increase in GLUT 1 immunoreactivity on the lumenal and ablumenal membrane as well as in the cytoplasm [62]. The findings in the rat and human diabetic retinal vessels appear at odds, but the duration of diabetes was so very different (a few weeks in the rats, over 15 years in the human subjects) that the findings could in fact describe different stages and influences in the development of the microangiopathy.

There is little information on whether and how retinal microvascular cells in situ are specifically damaged by hyperglycaemia. A progressive accumulation of advanced glycation end-products (AGEs) has been noted in the retinal vessels of rats who had diabetes for 2-8 months, with strong immunoreactivity localized around the pericyte nuclei [63]. The AGEs found in the retinal vessels of diabetic rats appear to be derived from both the oxidative and non-oxidative pathway of AGE formation [64] and there is no clear evidence for changes in the AGE receptors [63,64]. The retinal vessels of diabetic patients have been test- 
ed only for products of the oxidative pathway of AGE formation, which were found increased in the basement membranes of large vessels [43].

Increased haeme-oxygenase 1 immunostaining [65] and products of lipids peroxidation [66] in the retina of diabetic rats, as well as activation of the transcription factor nuclear factor $\varkappa \mathrm{B}(\mathrm{NF}-\varkappa \mathrm{B})$ [67] and up-regulated expression of glutathione peroxidase [29] in human diabetic pericytes suggest the occurrence of oxidant stress. A few studies have tested the effect of antioxidants on the characteristic vascular pathology of diabetic retinopathy. Neither nicartine [65] nor probuchol [68] administered to streptozotocin-diabetic rats for 6 months prevented the development of acellular capillaries. Nicartine, however, reduced pericyte loss by $40 \%$ [65] and a similar protective effect on pericytes was reported with trolox in rats with 5 months of diabetes [69]. It should be noted that not all investigators detect vascular histopathology in the rat retina after 5-6 months of diabetes [31], and therefore the results of these studies await confirmation at more advanced stages of the microangiopathy. Nonetheless, the observations are of interest from several standpoints. First, they indicate a greater susceptibility of pericytes than endothelial cells to oxidative stress, consistent with the finding that in the human diabetic retina NF- $x \mathrm{~B}$ activation was selectively observed in the pericytes [67]. Second, they suggest that oxidative stress is not the major driving force in the pathogenesis of diabetic microangiopathy. It has been pointed out that diabetes induces more of a "substrate" stress than an oxidant stress, because the increased generation of reactive carbonyl compounds by overloaded metabolic pathways occurs through both oxidative and non-oxidative routes [70] so that antioxidant therapy could miss a large fraction of the target [70]. This view would explain why aminoguanidine, which acts as a general carbonyl scavenger, trapping both oxidative and non-oxidative species derived from carbohydrate as well as lipid metabolism [70], is more effective than antioxidants in preventing the vascular lesions of diabetic retinopathy in rats $[68,71]$. On the other hand, it is possible that aminoguanidine acts through mechanisms other than, or in addition to, the prevention of AGE formation. The drug was recently reported to prevent vascular apoptosis and histopathology in experimental diabetic retinopathy without preventing the accumulation of several AGEs [72]. Hence, the capacity of aminoguanidine to target multiple processes could be at the basis of its preventative effects on both pericyte loss and the development of acellular capillaries, two events increasingly suspected to have a different pathogenesis.

Changes relevant to altered properties of the endothelial barrier. Normally, retinal and brain vascular endothelium functions as a selective barrier using three mechanisms: the presence of specific transporters or enzymes; minimal or absent pinocytosis which limits the transcellular passage of solutes; and continuous inter-endothelial tight junctions which prevent paracellular diffusion of solutes [52]. Increased endocytosis by retinal vascular endothelial cells has been observed in rats infused with horseradish peroxidase who have had diabetes for a short duration (6 weeks). However, the tracer present in endocytotic vesicles was not transported outside the endothelium [73]. A similar finding had been reported almost two decades previously in dogs who had had diabetes for 5 years [74]. In the diabetic dogs, the infused horseradish peroxidase was present in pinocytotic vesicles within the cytoplasm of retinal endothelium but did not identify leaky vessels [74]. At possible variance with these results in animal models, a recent study on the human diabetic retina has documented a spatial correlation between the vascular expression of the PAL-E antigen-which is associated with the endothelial plasmalemmal vesicles and normally absent from barrier endothelium-and leakage of plasma proteins [75]. Increased pinocytosis thus appears as an early and sustained abnormality of the retinal endothelial cells in diabetes which could or could not translate into increased permeability. On the other hand, diabetic retinal vessels clearly become abnormally permeable when tight junctions are altered [74]. Tight junctions seal the paracellular space through a continuous network of intermembrane fibrils formed by the transmembrane proteins claudins and occludin [76]. Occludin binds to the cytosolic plaque proteins $\mathrm{ZO}-1$ and $\mathrm{ZO}-2$, which, in turn, bind actin filaments, thus anchoring the tight junction to the cytoskeleton [76]. Occludin was the first integral transmembrane protein of tight junctions to be identified and its behaviour in diabetes has now been investigated in two studies. One study has reported a $35 \%$ reduction in retinal occludin content concomitant with an increased vascular leakage of fluoresceinated albumin in rats who have had streptozotocin diabetes for 3 months [29]. However, in retinas obtained postmortem from human subjects who had Type II diabetes for 5-10 years, and showing albumin permeation of the vascular walls, a fourfold increase in occludin content was detected [77]. There could be several reasons for this discrepancy, possibly including the short duration of diabetes in experimental rats when compared with the human subjects. Of note, overexpression of occludin in cultured epithelial cells might increase on the one hand transepithelial electrical resistance (an index of the strength of the tight junction seal), but is on the other hand associated with increased paracellular flux [78]. If this scenario pertains to occludin function in vascular endothelium, occludin overexpression could affect the physiology and permeability of retinal capillary tight junctions as much as occludin underexpression. Much additional 
work is clearly required to ascertain how the molecular composition of tight junctions is altered in diabetes and which changes are necessary and sufficient to compromise the seal.

Changes relevant to capillary obliteration. Ultimately, retinal capillaries in diabetes become acellular and non-perfused, tubes enclosed by thickened basement membranes and filled with debris and glial processes [54, 74]. A molecular abnormality immediately relevant to these morphological changes is the up-regulated synthesis of basement membrane components. When compared with non-diabetic human donors, diabetic donors show increased retinal levels of the mRNAs encoding collagen IV [79], the predominant structural component of basement membranes, and fibronectin [80], an extrinsic component of basement membranes especially concentrated at pericyte-endothelial contacts [81] and increased in human retinal microvessels [80]. The retinal vessels of patients with early stages of diabetic retinopathy show additionally an increase in integrin $\beta 1$ [82], the subunit common to the family of integrin heterodimers that function as receptors for matrix molecules. This indicates that the processes contributing to basement membrane thickening are accompanied by modifications of the relation between cells and their matrix. Whether these modifications impact on the function of retinal microvascular cells in diabetes has not been resolved. In vitro studies have shown that excess extracellular matrix leads to increased cell-matrix adhesion and cytoskeletal rearrangements, eventually hampering endothelial cell replication [83], a series of events that, if occurring in vivo, could undermine the anti-thrombogenic properties of retinal microvascular endothelium. Other changes described in diabetic retinal vessels are poised to undermine such properties in a more direct manner. The combination of reduced expression of tissue plasminogen activator [84] and increased expression of plasminogen activator inhibitor-1 [85] observed in the retinal vessels of human diabetic donors could severely impair fibrinolysis. Increased expression of intercellular adhesion molecule-1 (ICAM-1) [86] could also confer to the endothelium pro-inflammatory properties. However, retinal vessels of diabetic rats also express more prostacyclin-stimulating factor [25], and produce more prostacyclin [87], an inhibitor of platelet aggregation and platelet adherence to the endothelium, as well as a potent vasodilator. These changes have been tested for relevance to retinal haemodynamics in diabetes [25] but can be expected to influence as well the functional properties of the luminal surface of diabetic vessels.

In both human and experimental diabetes retinal vascular cells die prematurely and show biochemical and morphological features consistent with apoptosis
[28]. Pericyte apoptosis is more readily detectable than endothelial cell apoptosis, most probably because the pericytes are encased in basement membrane and thus less accessible to clearing mechanisms, whereas apoptotic endothelial cells slough off into the capillary lumen and are cleared by blood flow. Microvascular cell apoptosis as detected by the terminal deoxynucleotide transferase (TUNEL) reaction is not yet evident in rats who have had diabetes for 2 or 5 months [33], but becomes evident after 7-8 months [31], and precedes the appearance of vascular histopathology [31]. Investigations on the molecular mechanisms underlying the accelerated apoptosis have assessed whether diabetes changes the levels, and thus the balance, of endogenous regulators of apoptosis. In the adult human retina, Bcl-2, the best known pro-survival member of the Bcl-2 family of endogenous regulators of apoptosis, was found almost exclusively in Müller glial cells, but could not be detected in vessels (Fig.2), and its abundance was not modified by diabetes [55]. Similarly, the retinal content of the pro-survival $\left(\mathrm{Bcl}-\mathrm{X}_{\mathrm{L}}\right)$ and proapoptotic $\left(\mathrm{Bcl}-\mathrm{X}_{\mathrm{S}}\right)$ isoforms of $\mathrm{Bcl}-\mathrm{X}$ was not altered by diabetes [47]. Diabetes did instead increase the content of proapoptotic Bax [47] and in retinal microvessels intense Bax staining was often detected around the fragmented (apoptotic) nuclei of pericytes, indicating that increased Bax expression is related, at least temporally, to the accelerated death of pericytes. As noted earlier for NF- $x \mathrm{~B}$ activation [67], increased Bax was observed selectively in the pericytes and no staining was detected in endothelial cells. Hence, the aggregate of molecular changes noted in diabetic pericytes (Table 1) suggests a pathway to apoptosis that involves oxidant stress and Bax up regulation, which can be triggered by high glucose [47]. In contrast, the molecular changes attributable to the endothelium are indicative rather of an activated phenotype that could contribute to apoptosis through indirect mechanisms such as heightened interactions with circulating elements. Further investigation is required to assess the nature and mechanisms of retinal endothelial cell activation in diabetes, and whether it coexists with endothelial-intrinsic pathways to apoptosis which have not yet been revealed by studies done to date.

\section{Multiple processes could contribute to retinal diabetic microangiopathy}

The cellular and molecular abnormalities described above begin to reveal processes operative in the diabetic retina. The following is a synthetic view of how multiple abnormalities could converge in the causation of the two clinically important features of diabetic microangiopathy, increased capillary permeability and capillary occlusion and obliteration. 
Increased capillary permeability. Increased endocytosis $[73,75]$ and altered content of the tight junction protein occludin $[29,77]$ point to an endothelial cell phenotype that has lost some of its barrier features. The inciting events could originate within and/or outside the endothelial cells. Diabetes could act on endothelial cells to change directly how they regulate transcellular transport or synthesize and assemble tight junction proteins, but such effects have not yet been demonstrated experimentally. The up-regulated expression of proinflammatory molecules found on retinal endothelial cells in diabetes could be a mechanism for capillary leakage through the induction of leukostasis $[86,88]$. And later in the course of diabetes, the accelerated death [31] and turnover [89] of retinal endothelial cells could compromise the timely acquisition of the highly differentiated barrier phenotype. This could in turn be compounded by altered capability of the glial cells affected by diabetes [28, $55,56]$ to provide their inductive influences on barrier endothelium. Increased expression of VEGF by the neuroretina-when occurring [24]-can be expected to alter endothelial cell properties so as to increase permeability; and, finally, the activated monocytes and granulocytes encountered in diabetes [90, 91] could contribute to vascular leakage through nonspecific damage to the endothelium. This constellation of abnormalities indicates that the increased permeability of retinal vessels in diabetes is a multifactorial process, where the relative importance of individual contributors could change during the course of diabetes, and could differ in different individuals. Hence, the perspective within which studies are interpreted should be wide and inclusive.

Capillary occlusion and obliteration. A similarly inclusive approach appears to be desirable in reconstructing the process(es) underlying capillary occlusion and obliteration. The documentation of reperfusion of retinal capillaries in diabetic patients studied longitudinally [92] indicates that occlusive events are, at least for some time, reversible. In the diabetic rat model, adhesion of leukocytes to retinal capillaries has been noted within days of the induction of diabetes $[88,91]$, mediated by an increased expression of both endothelial ICAM-1 [88] and neutrophil integrins [91]. Later on, in the course of experimental [93] or spontaneous [94] diabetes in the rat, retinal capillaries are found occluded by platelet-fibrin thrombi, surrounded by leukocytes and erythrocytes. Recently, increased prevalence of microthromboses has also been quantitatively demonstrated in the retinal vessels of diabetic patients [95]. When compared with those of non-diabetic donors, the retinas of diabetic donors showed twice the number of capillaries with fibrin-platelet thrombi, and a four-fold increase in the total area of capillary segments occupied by thrombi. In both the diabetic and non-diabetic sub- jects, there was a significant topographical association of microthrombosis with apoptotic vascular cells [95]. Microthrombosis and apoptosis probably have a circular relation because platelet aggregation can damage microvascular endothelium causing ischaemia/reperfusion, while apoptotic endothelial cells can become procoagulant [96] and hyperadhesive [97] and incite microthrombosis. The fact that apoptosis and microthrombosis become detectable in experimental animals after several months of diabetes $[31,93,94]$ suggests that the risk increases over time, a kinetic pattern consistent with the occurrence of cumulative vascular damage. An intriguing question is whether some of the cumulative damage reflects the cumulative effects of high glucose. This could be the case in pericytes, which accelerate their rate of apoptosis after several weeks of exposure to high glucose in vitro [47]. No data are available on the susceptibility of retinal endothelial cells to the ill effects of high glucose, and it will be a challenge to address such an issue in vitro. At variance with pericytes, retinal endothelial cells show in vitro exuberant proliferation, a state that is both fundamentally different from the replicative quiescence observed in retinal vessels [98] and incompatible with the very goal of testing cumulative effects of high glucose on a stable cell population.

The consequences of retinal microvascular cell apoptosis can readily account for many features of diabetic retinopathy. Pericyte apoptosis is the probable prodrome of pericyte "drop out" and "ghosts" because pericytes regenerate minimally, if at all, in the adult retina [98]. Endothelial cell apoptosis can initiate the accelerated turnover of endothelial cells [89], which in turn can contribute to the accumulation and layering of basal membranes, as well as to vascular remodelling. The life cycle of endothelial cells can be a hidden arbiter of the inertia with which retinal vessels respond to changes in metabolic control [2, 99, 100]. Accelerated apoptosis and turnover during poor metabolic control are likely to deplete the replicative reserve of endothelial cells, and this could account for the progression of diabetic microangiopathy from apparently normal vessels and despite restoration of normoglycaemia [99, 100]. Conversely, the spared replicative reserve of retinal endothelial cells during tight metabolic control could be a capital that lessens, for some time, the impact of later demands. This concept could be applicable to the observation that the intensely-treated DCCT cohort experiences in follow-up persistent protection from progression of retinopathy despite a return to increasing hyperglycaemia [2]. The slow accumulation and long half-life of AGEs have been invoked to explain the delay with which retinopathy responds to prevailing glycaemic levels [2], but it is of note that the development of vascular histopathology in two animal models of diabetic retinopathy was predicted 
by the rate of microvascular cell apoptosis and had no correlation with the accumulation of several AGEs [72].

The three processes that can currently be associated with capillary occlusion and obliteration-proinflammatory changes, apoptosis, and microthrombosis-have been documented in human diabetic retinal vessels [31, 86, 95]. It is thus tempting to view such processes as targets for available drugs. The recent demonstration that aspirin administered to dogs from the onset of experimental diabetes prevented the development of acellular capillaries [101], reawakens interest in past studies showing the beneficial effect of aspirin or other anti-platelet agents on blood flow [102] and development of microaneurysms [103, $104]$ in patients with early diabetic retinopathy.

\section{Perspectives and caveats}

Ten years ago, in a Perspective in Diabetes, we drew attention to the importance of ascertaining "whether and how the cells involved in the complications of diabetes modify their differentiated functions in vivo", through the application of the then novel techniques to study gene expression in situ in complex organs [105]. As shown by this review, much work has been done, but more is needed to arrive at a cohesive description of the pathogenesis and pathophysiology of diabetic retinopathy. We expect that future work will develop the promising leads obtained to date, and uncover new ones using the latest generation of molecular and genetic approaches. Microarray technology, optimally combined with methods to isolate specific populations of retinal cells, will provide global profiles of the changes in gene expression induced by diabetes. A recent study comparing normal and tumor endothelium [106] is a beautiful example of the type of work that could tell us comprehensively and without bias about endothelial changes in diabetic retinopathy. Comprehensive monitoring of gene expression will also unravel the nature of the retinal "neuropathy" and "gliopathy" that diabetes seems to cause. The use of genetically engineered animals will help investigators define the mechanistic role of candidate abnormalities, and thus guide studies on prevention and treatment. A third new tool, the sequence of the human genome, could become instrumental in exploring genetic contributions to diabetic retinopathy although the rationale for undertaking genome-wide screens would have to be more firmly established.

The potential of these new approaches must contend with the limitations of the specimens and models to which they can be applied. Work on the human diabetic retina continues to provide an essential sounding board in the field but requires precautions and has intrinsic limitations. The retinas are obtained postmortem and thus from elderly individuals who are affected often by other pathologies in addition to diabetes. The potentially confounding influences imposed by these characteristics can be ascertained by appropriate experiments in animals $[33,40]$ and controlled at least in part by precise inclusion and exclusion criteria as well as by rigorous matching of the characteristics of diabetic and non-diabetic donors. However, the fact remains that postmortem human retinas do not permit studies of signal transduction [33] and will mostly manifest the effects of Type II diabetes, which might or might not be identical to those of Type I diabetes. Streptozotocin-diabetic rats have shed much light on early retinal microangiopathy because they develop several of the characteristic lesions known in human diabetic retinopathy, but require further evaluation as a model for neuroglial dysfunction. Acute streptozotocin toxicity is probably not a confounding factor because the neuroglial abnormalities reported in streptozotocin-diabetic rats were prevented by intensive insulin treatment $[12,14,30]$, noted to occur over time $[23,28,33,56$, 57], and/or also shown in spontaneously diabetic rats or humans $[15,33,57]$. However, prevention by intensive insulin and time-related changes cannot rule out contributions to neuroglial abnormalities from the low growth hormone levels [38] and low thyroid state [107] that accompany severe diabetes in the streptozotocin-diabetic rats. Minimal precautions will be to study animals that, even in the short-term, receive sufficient maintenance insulin to prevent weight loss and hypothyroidism and to seek verification of critical findings in the human diabetic retina. Finally, overexpression or targeted deletion of candidate genes in vivo are generally engineered in mice and mice are not yet well established models for the study of retinopathy. Mice have been shown to develop the microvascular lesions characteristic of human nonproliferative diabetic retinopathy but the lesions were induced by galactose feeding and more that 15 months were required for their number to be significantly greater than in control mice [108]. Because the pathogenesis of diabetic and galactose-induced retinopathy differ at an early stage [72], galactosemic mice might not be an appropriate model. Consideration should also be given to the spontaneous pathologies that specific strains of mice develop and that are bound to interfere with the study of the effects of diabetes. For example, the FVB mouse strain, which is used extensively in transgenic research because of its well-defined inbred background and easiness of DNA microinjection in the fertilized zygote, develops early retinal degeneration on account of the $r d / r d$ genotype [109]. The need for better characterized and innovative mouse and non-mouse models to study retinopathy and other diabetic complications is acknowledged in recent requests for grant applications (RFA) by the United States National Institutes of Health (RFA DK-01-009 and HL-01-010). 
Diabetic retinopathy is a difficult process to investigate owing to its protracted course and multiple endpoints and these features will always be a hurdle even as we begin to understand its biology and have access to ever more powerful technology. The good news, however, is that we are in a position to stall the process by helping our patients achieve good metabolic and hypertension control, while we continue to labor at the scientific issues and at the next rational intervention.

Acknowledgements. Cited work from the authors' laboratory was supported by grants from the United States Public Health Service (EY-09122) and the American Diabetes Association, a Career Development Award from the Juvenile Diabetes Foundation International (2-2000-357, to CG), and the George and Frances Levin Endowment. The review is based on the relevant literature published in the English language during the period 1990-2001, and seminal prior contributions. The sources available to the authors were integrated with sources identified through PubMed searches for "neural retina and diabetes", "retina glia and diabetes", and "retinal vessels and diabetes".

\section{References}

1. Pirart J (1978) Diabetes mellitis and its degenerative complications: A prospective study of 4,400 patients observed between 1947 and 1973. Diabetes Care 1: 168-188

2. The Diabetes Control and Complications Trial/Epidemiology of Diabetes Interventions and Complications Research Group (2000) Retinopathy and nephropathy in patients with type 1 diabetes four years after a trial of intensive therapy. N Engl J Med 342: 381-389

3. UK Prospective Diabetes Study Group (1998) Tight blood pressure control and risk of macrovascular and microvascular complications in type 2 diabetes: UKPDS 38. BMJ 317: 703-713

4. Klein R, Klein BEK, Moss SE, Davis MD, DeMets DL (1984) The Wisconsin epidemiologic study of diabetic retinopathy. II. Prevalence and risk of diabetic retinopathy when age at diagnosis is less than 30 years. Arch Ophthalmol 102: 520-526

5. Klein R, Klein BEK, Moss SE, Davis MD, DeMets DL (1984) The Wisconsin epidemiologic study of diabetic retinopathy. III. Prevalence and risk of diabetic retinopathy when age at diagnosis is 30 or more years. Arch Ophthalmol 102: 527-532

6. Simonsen SE (1968) ERG in diabetics. In: The clinical value of electroretinography, ISCERG Symp. Ghent 1966, Karger, Basel/New York pp 403-412

7. Ghirlanda G, Di Leo MAS, Caputo S, Cercone S, Greco AV (1997) From functional to microvascular abnormalities in early diabetic retinopathy. Diabetes Metab Rev 13: $15-35$

8. Parisi V, Uccioli L, Parisi L et al. (1998) Neural conduction in visual pathways in newly-diagnosed IDDM patients. Electroencephalogr Clin Neurophysiol 108: 490-496

9. MacNeil MA, Masland RH (1998) Extreme diversity among amacrine cells: Implications for function. Neuron 20: 971-982

10. Petters RM, Alexander CA, Wells KD et al. (1997) Genetically engineered large animal model for studying cone photoreceptor survival and degeneration in retinitis pigmentosa. Nat Biotechnol 15: 965-970

11. Darius S, Wolf G, Huang PL, Fishman MC (1995) Localization of NADPH-diaphorase/nitric oxide synthase in the rat retina: an electron microscopic study. Brain Res 690: 231-235

12. Roufail E, Soulis T, Boel E, Cooper ME, Rees S (1998) Depletion of nitric oxide synthase-containing neurons in the diabetic retina: reversal by aminoguanidine. Diabetologia 41: 1419-1425

13. Deng D, Evans T, Mukherjee K, Downey D, Chakrabarti $S$ (1999) Diabetes-induced vascular dysfunction in the retina: role of endothelins. Diabetologia 42: 1228-1234

14. De Juan JA, Moya FJ, Ripodas A, Bernal R, FernandezCruz A, Fernandez-Durango R (2000) Changes in the density and localisation of endothelin receptors in the early stages of rat diabetic retinopathy and the effect of insulin treatment. Diabetologia 43: 773-785

15. Chakrabarti S, Gan XT, Merry A, Karmazyn M, Sima AAF (1998) Augmented retinal endothelin-1, endothelin-3, endothelin A $_{\mathrm{A}}$ and endothelin $\mathrm{B}_{\mathrm{B}}$ gene expression in chronic diabetes. Curr Eye Res 17: 301-307

16. Takagi C, Bursell SE, Lin YW et al. (1996) Regulation of retinal hemodynamics in diabetic rats by increased expression and action of endothelin-1. Invest Ophthalmol Vis Sci 37: 2504-2518

17. De La Rubia G, Oliver FJ, Inoguchi T, King GL (1992) Induction of resistance to endothelin-1's biochemical actions by elevated glucose levels in retinal pericytes. Diabetes 41: 1533-1539

18. Evans T, Deng DX, Chen S, Chakrabarti S (2000) Endothelin receptor blockade prevents augmented extracellular matrix component mRNA expression and capillary basement membrane thickening in the retina of diabetic and galactose-fed rats. Diabetes 49: 662-666

19. Ishii H, Jirousek MR, Koya D et al. (1996) Amelioration of vascular dysfunctions in diabetic rats by an oral $\mathrm{PKC} \beta$ inhibitor. Science 272: 728-731

20. Badr GA, Tang J, Ismail-Beigi F, Kern TS (2000) Diabetes downregulates GLUT1 expression in the retina and its microvessels but not in the cerebral cortex or its microvessels. Diabetes 49: 1016-1021

21. Kumagai AK (1999) Glucose transport in brain and retina: Implications in the management and complications of diabetes. Diabetes Metab Res Rev 15: 261-273

22. Pekala P, Marlow M, Heuveiman D, Connolly D (1990) Regulation of hexose transport in aortic endothelial cells by vascular permeability factor and tumor necrosis factor- $\alpha$, but not by insulin. J Biol Chem 265: 18051-18054

23. Murata T, Nakagawa K, Khalil A, Ishibashi T, Inomata H, Sueishi K (1996) The relation between expression of vascular endothelial growth factor and breakdown of the blood-retinal barrier in diabetic rat retinas. Lab Invest 74: 819-825

24. Hammes, H-P, Lin J, Bretzel RG, Brownlee M, Breier G (1998) Upregulation of the vascular endothelial growth factor/vascular endothelial growth factor receptor system in experimental background diabetic retinopathy of the rat. Diabetes 47: 401-406

25. Hata Y, Clermont A, Yamauchi T et al. (2000) Retinal expression, regulation, and functional bioactivity of prostacyclin-stimulating factor. J Clin Invest 106: 541-550

26. Ferrara N, Davis-Smyth T (1997) The biology of vascular endothelial growth factor. Endocr Rev 18: 4-25

27. Tolentino MJ, Miller JW, Gragoudas ES et al. (1996) Intravitreous injections of vascular endothelial growth fac- 
tor produce retinal ischemia and microangiopathy in an adult primate. Ophthalmology 103: 1820-1828

28. Rungger-Brändle E, Dosso AA, Leuenberger PM (2000) Glial reactivity, an early feature of diabetic retinopathy. Invest Ophthalmol Vis Sci 41: 1971-1980

29. Antonetti DA, Barber AJ, Khin S et al. (1998) Vascular permeability in experimental diabetes is associated with reduced endothelial occludin content. Vascular endothelial growth factor decreases occludin in retinal endothelial cells. Diabetes 47: 1953-1959

30. Barber AJ, Lieth E, Khin SA et al. (1998) Neural apoptosis in the retina during experimental and human diabetes. Early onset and effect of insulin. J Clin Invest 102: 783-791

31. Mizutani M, Kern TS, Lorenzi M (1996) Accelerated death of retinal microvascular cells in human and experimental diabetic retinopathy. J Clin Invest 97: 2883-2890

32. Li W, Yanoff M, Jian B, He Z (1999) Altered mRNA levels of antioxidant enzymes in pre-apoptotic pericytes from human diabetic retinas. Cell Mol Biol 45: 59-66

33. Gerhardinger C, McClure KD, Romeo G, Podestà F, Lorenzi $M$ (2001) IGF-I mRNA and signaling in the diabetic retina. Diabetes 50: 175-183

34. Peruzzi F, Prisco M, Dews M et al. (1999) Multiple signaling pathways of the insulin-like growth factor 1 receptor in protection from apoptosis. Mol Cell Biol 19: 7203-7215

35. King GL, Goodman AD, Buzney S, Moses A, Kahn CR (1985) Receptors and growth-promoting effects of insulin and insulin-like growth factors on cells from bovine retinal capillaries and aorta. J Clin Invest 75: 1028-1036

36. Burren CP, Berka JL, Edmondson SR, Werther GA, Batch JA (1996) Localization of mRNAs for insulin-like growth factor-I (IGF-I), IGF-I receptor, and IGF binding proteins in rat eye. Invest Ophthalmol Vis Sci 37: 1459-1468

37. Bach LA, Rechler MM (1992) Insulin-like growth factors and diabetes. Diabetes Metab Rev 8: 229-257

38. Fagin JA, Roberts CT, Le Roith D, Brown AT (1989) Coordinate decrease of tissue insulin-like growth factor I post-transcriptional alternative mRNA transcripts in diabetes mellitus. Diabetes 38: 428-434

39. Kumagai AK, Glasgow BJ, Pardridge WM (1994) GLUT1 glucose transporter expression in the diabetic and nondiabetic human eye. Invest Ophthalmol Vis Sci 35: 2887-2894

40. Gerhardinger C, Brown LF, Roy S, Mizutani M, Zucker CL, Lorenzi M (1998) Expression of vascular endothelial growth factor in the human retina and in nonproliferative diabetic retinopathy. Am J Pathol 152: 1453-1462

41. Amin RH, Frank RN, Kennedy A, Eliott D, Puklin JE, Abrams GW (1997) Vascular endothelial growth factor is present in glial cells of the retina and optic nerve of human subjects with nonproliferative diabetic retinopathy. Invest Ophthalmol Vis Sci 38: 36-47

42. Mathews MK, Merges C, McLeod DS, Lutty GA (1997) Vascular endothelial growth factor and vascular permeability changes in human diabetic retinopathy. Invest Ophthalmol Vis Sci 38: 2729-2741

43. Murata T, Nagai R, Ishibashi T, Inomata H, Ikeda K, Horiuchi $S$ (1997) The relationship between accumulation of advanced glycation end products and expression of vascular endothelial growth factor in human diabetic retinas. Diabetologia 40: 764-769

44. Miller JW, Adamis AP, Shima DT et al. (1994) Vascular endothelial growth factor/vascular permeability factor is temporally and spatially correlated with ocular angiogenesis in a primate model. Am J Pathol 145: 574-584
45. Kim I, Ryan AM, Rohan R et al. (1999) Constitutive expression of VEGF, VEGFR-1, and VEGFR-2 in normal eyes. Invest Ophthalmol Vis Sci 40: 2115-2121

46. Holländer H, Makarov F, Dreher Z, van Driel D, ChanLing T, Stone J (1991) Structure of the macroglia of the retina: Sharing and division of labour between astrocytes and Müller cells. J Comp Neurol 313: 587-603

47. Podestà F, Romeo G, Liu W-H et al. (2000) Bax is increased in the retina of diabetic subjects and is associated with pericyte apoptosis in vivo and in vitro. Am J Pathol 156: 1025-1032

48. Poitry-Yamate CL, Poitry S, Tsacopoulos M (1995) Lactate released by Müller glial cells is metabolized by photoreceptors from mammalian retina. $\mathrm{J}$ Neurosci 15: 5179-5191

49. Ludvigson MA, Sorenson RL (1980) Immunohistochemical localization of aldose reductase. II. Rat eye and kidney. Diabetes 29: 450-459

50. Akagi Y, Yajima Y, Kador PF, Kuwabara T, Kinoshita JH (1984) Localization of aldose reductase in the human eye. Diabetes 33: 562-566

51. Newman E, Reichenbach A (1996) The Müller cell: a functional element of the retina. Trends Neurosci 19: 307-312

52. Risau W (1991) Induction of blood-brain barrier endothelial cell differentiation. Ann NY Acad Sci 633: 405-419

53. Tout S, Chan-Ling T, Holländer H, Stone J (1993) The role of Müller cells in the formation of the blood-retinal barrier. Neuroscience 55: 291-301

54. Bek T (1997) Glial cell involvement in vascular occlusion of diabetic retinopathy. Acta Ophthalmol Scand 75: 239-243

55. Mizutani M, Gerhardinger C, Lorenzi M (1998) Müller cell changes in human diabetic retinopathy. Diabetes 47 : 445-449

56. Lieth E, Barber AJ, Xu B et al. (1998) Glial reactivity and impaired glutamate metabolism in short-term experimental diabetic retinopathy. Diabetes 47: 815-820

57. Barber AJ, Antonetti DA, Gardner TW, and the Penn State Retina Research Group (2000) Altered expression of retinal occludin and glial fibrillar acidic protein in experimental diabetes. Invest Ophthalmol Vis Sci 41: 3561-3568

58. Messing A, Head MW, Galles K, Galbreath EJ, Goldman JE, Brenner M (1998) Fatal encephalopathy with astrocyte inclusions in GFAP transgenic mice. Am J Pathol 152: 391-398

59. Eddleston M, Mucke L (1993) Molecular profile of reactive astrocytes-implications for their role in neurologic disease. Neuroscience 54: 15-36

60. Ennis SR, Johnson JE, Pautler EL (1982) In situ kinetics of glucose transport across the blood-retinal barrier in normal rats and rats with streptozocin-induced diabetes. Invest Ophthalmol Vis Sci 23: 447-456

61. Howard RL (1996) Down-regulation of glucose transport by elevated extracellular glucose concentrations in cultured rat aortic smooth muscle cells does not normalize intracellular glucose concentrations. J Lab Clin Med 127: 504-515

62. Kumagai AK, Vinores SA, Pardridge WM (1996) Pathological upregulation of inner blood-retinal barrier Glut 1 glucose transporter expression in diabetes mellitus. Brain Research 706: 313-317

63. Stitt AW, Li YM, Gardiner TA, Bucala R, Archer DB, Vlassara H (1997) Advanced glycation end products (AGEs) co-localize with AGE receptors in the retinal vasculature of diabetic and of AGE-infused rats. Am J Pathol 150: 523-531 
64. Hammes H-P, Alt A, Niwa T et al. (1999) Differential accumulation of advanced glycation end products in the course of diabetic retinopathy. Diabetologia 42: 728-736

65. Hammes HP, Bartmann A, Engel L, Wülfroth P (1997) Antioxidant treatment of experimental diabetic retinopathy in rats with nicanartine. Diabetologia 40: 629-634

66. Kowluru RA, Kern TS, Engerman RL, Armstrong D (1996) Abnormalities of retinal metabolism in diabetes or experimental galactosemia. III. Effects of antioxidants. Diabetes 45: 1233-1237

67. Lorenzi M, Gerhardinger C (2000) Pathophysiology of diabetic retinopathy. In: DiMario U, Leonetti F, Pugliese G, Sbraccia P, Signore A (eds) Diabetes in the new millennium. Wiley, Chichester, pp 287-296

68. Agardh E, Hultberg B, Agardh CD (2000) Effects of inhibition of glycation and oxidative stress on the development of cataract and retinal vessels abnormalities in diabetic rats. Curr Eye Res 21: 543-549

69. Ansari NH, Zhang W, Fulep E (1998) Prevention of pericyte loss by trolox in diabetic rat retina. J Toxicol Environ Health 54: 467-475

70. Baynes JW, Thorpe SR (1999) Role of oxidative stress in diabetic complications. A new perspective on an old paradigm. Diabetes 48: 1-9

71. Hammes H-P, Martin S, Federlin K et al. (1991) Aminoguanidine treatment inhibits the development of experimental diabetic retinopathy. Proc Natl Acad Sci USA 88: $11555-11558$

72. Kern TS, Tang J, Mizutani M et al. (2000) Response of capillary cell death to aminoguanidine predicts the development of retinopathy: Comparison of diabetes and galactosemia. Invest Ophthalmol Vis Sci 41: 3972-3978

73. Gardiner TA, Stitt AW, Archer DB (1995) Retinal vascular endothelial cell endocytosis increases in early diabetes. Lab Invest 72: 439-444

74. Wallow IHL, Engerman RL (1977) Permeability and patency of retinal blood vessels in experimental diabetes. Invest Ophthalmol Vis Sci 16: 447-461

75. Schlingemann RO, Hofman P, Vrensen GFJM, Blaauwgeers HGT (1999) Increased expression of endothelial antigen PAL-E in human diabetic retinopathy correlates with microvascular leakage. Diabetologia 42: 596-602

76. Fanning AS, Mitic LL, Anderson JM (1999) Transmembrane proteins in the tight junction barrier. J Am Soc Nephrol 10: 1337-1345

77. Gerhardinger C, McClure KD, Lorenzi M (2000) Mechanisms of occludin overexpression in human diabetic retinopathy. Diabetes 49 [Suppl 1]:A170 (Abstract)

78. Balda MS, Whitney JA, Flores C, González S, Cereijido M, Matter K (1996) Functional dissociation of paracellular permeability and transepithelial electrical resistance and disruption of the apical-basolateral intramembrane diffusion barrier by expression of a mutant tight junction membrane protein. J Cell Biol 134: 1031-1049

79. Roy S, Maiello M, Lorenzi M (1994) Increased expression of basement membrane collagen in human diabetic retinopathy. J Clin Invest 93: 438-442

80. Roy S, Cagliero E, Lorenzi M (1994) Fibronectin overexpression in retinal microvessels of patients with diabetes. Invest Ophthalmol Vis Sci 37: 258-266

81. Courtoy PJ, Boyles J (1983) Fibronectin in the microvasculature: Localization in the pericyte-endothelial interstitium. J Ultrastruct Res 83: 258-273

82. Roth T, Podestá F, Stepp MA, Boeri D, Lorenzi M (1993) Integrin overexpression induced by high glucose and by human diabetes: Potential pathway to cell dysfunction in diabetic microangiopathy. Proc Natl Acad Sci USA 90: 9640-9644

83. Podestá F, Roth T, Ferrara F, Cagliero E, Lorenzi M (1997) Cytoskeletal changes induced by excess extracellular matrix impair endothelial cell replication. Diabetologia 40: 879-886

84. Lutty GA, Ikeda K, Chandler C, McLeod DS (1991) Immunolocalization of tissue plasminogen activator in the diabetic and nondiabetic retina and choroid. Invest Ophthalmol Vis Sci 32: 237-245

85. Cagliero E, Grant MB, Lorenzi M (1991) Measurement of gene expression in human retinal microvessels by solution hybridization. Invest Ophthalmol Vis Sci 32: $1439-1445$

86. McLeod DS, Lefer DJ, Merges C, Lutty GA (1995) Enhanced expression of intracellular adhesion molecule-1 and $\mathrm{P}$-selectin in the diabetic human retina and choroid. Am J Pathol 147: 642-653

87. Johnson EIM, Dunlop ME, Larkins RG (1999) Increased vasodilatory prostaglandin production in the diabetic rat retinal vasculature. Curr Eye Res 18: 79-82

88. Miyamoto, K, Khosrof S, Bursell S-E et al. (1999) Prevention of leukostasis and vascular leakage in streptozotocininduced diabetic retinopathy via intercellular adhesion molecule-1 inhibition. Proc Natl Acad Sci USA 96: 10836-10841

89. Sharma NK, Gardiner TA, Archer DB (1985) A morphologic and autoradiographic study of cell death and regeneration in the retinal microvasculature of normal and diabetic rats. Am J Ophthalmol 100: 51-60

90. Schröder S, Palinksi W, Schmid-Schönbein GW (1991) Activated monocytes and granulocytes, capillary nonperfusion, and neovascularization in diabetic retinopathy. Am J Pathol 139: 81-100

91. Barouch FC, Miyamoto K, Allport JR et al. (2000) Integrin-mediated neutrophil adhesion and retinal leukostasis in diabetes. Invest Ophthalmol Vis Sci 41: 1153-1158

92. Takahashi K, Kishi S, Muraoka K, Shimizu K (1998) Reperfusion of occluded capillary beds in diabetic retinopathy. Am J Ophthalmol 126: 791-797

93. Ishibashi T, Tanaka K, Taniguchi Y (1981) Platelet aggregation and coagulation in the pathogenesis of diabetic retinopathy in rats. Diabetes 30: 601-606

94. Sima AAF, Chakrabarti S, Garcia-Salinas R, Basu PK (1985) The BB-rat - an authentic model of human diabetic retinopathy. Curr Eye Res 4: 1087-1092

95. Boeri D, Maiello M, Lorenzi M (2001) Increased prevalence of microthromboses in retinal capillaries of diabetic individuals. Diabetes 50: 1432-1439

96. Bombeli T, Karsan A, Tait JF, Harlan JM (1997) Apoptotic vascular endothelial cells become procoagulant. Blood 89: 2429-2442

97. Hébert M-J, Gullans SR, Mackenzie HS, Brady HR (1998) Apoptosis of endothelial cells is associated with paracrine induction of adhesion molecules. Evidence for an interleukin- $1 \beta$-dependent paracrine loop. Am J Pathol 152: 523-532

98. Engerman RL, Pfaffenbach D, Davis MD (1967) Cell turnover of capillaries. Lab Invest 17: 738-743

99. Engerman RL, Kern TS (1987) Progression of incipient diabetic retinopathy during good glycemic control. Diabetes 36: 808-812

100. Su EN, Alder VA, Yu DY, Yu PK, Cringle SJ, Yogesan K (2000) Continued progression of retinopathy despite spontaneous recovery to normoglycemia in a long-term study of streptozotocin-induced diabetes in rats. Graefes Arch Clin Exp Ophthalmol 238: 163-173 
101. Kern TS, Engerman RL (2000) Pharmacologic inhibition of diabetic retinopathy: Comparison of aminoguanidine and aspirin. Invest Ophthalmol Vis Sci 41 [Suppl 1]:S114 (Abstract)

102. Feke GT, Yoshida A, Ogasawara H et al. (1996) Retinal blood flow increases following short-term aspirin usage in type 1 diabetics with no or minimal retinopathy. Ophthalmic Res 28: 108-116

103. The Damad Study Group (1989) Effect of aspirin alone and aspirin plus dipyridamole in early diabetic retinopathy. A multicenter randomized controlled clinical trial. Diabetes 38: 491-498

104. The Timad Study Group (1990) Ticlopidine treatment reduces the progression of nonproliferative diabetic retinopathy. Arch Ophthalmol 108: 1577-1583
105. Lorenzi M, Cagliero E (1991) Pathobiology of endothelial and other vascular cells in diabetes mellitus. Call for data. Diabetes 40: 653-659

106. St. Croix B, Rago C, Velculescu V et al. (2000) Genes expressed in human tumor endothelium. Science 289: 1197-1202

107. Bestetti GE, Reymond MJ, Boujon CE, Lemarchand-Beraud, T, Rossi, GL (1989) Functional and morphological aspects of impaired TRH release by mediobasal hypothalamus of STZ-induced diabetic rats. Diabetes 38: 1351-1356

108. Kern TS, Engerman RL (1996) A mouse model of diabetic retinopathy. Arch Ophthalmol 114: 986-990

109. Griep AE, Krawcek J, Lee D et al. (1998) Multiple genetic loci modify risk for retinoblastoma in transgenic mice. Invest Ophthalmol Vis Sci 39: 2723-2732 